\title{
Article \\ Meander-DGS Effect on Electromagnetic Bandgap Structure for Power/Ground Noise Suppression in High-Speed Integrated Circuit Packages and PCBs
}

\author{
Myunghoi Kim (D)
}

check for

updates

Citation: Kim, M. Meander-DGS

Effect on Electromagnetic Bandgap Structure for Power/Ground Noise Suppression in High-Speed Integrated Circuit Packages and PCBs. Electronics 2022, 11, 211. https://doi.org/10.3390/ electronics11020211

Academic Editor: Gerardo Di Martino

Received: 23 November 2021 Accepted: 8 January 2022

Published: 10 January 2022

Publisher's Note: MDPI stays neutral with regard to jurisdictional claims in published maps and institutional affiliations.

Copyright: (C) 2022 by the author. Licensee MDPI, Basel, Switzerland. This article is an open access article distributed under the terms and conditions of the Creative Commons Attribution (CC BY) license (https:// creativecommons.org/licenses/by/ $4.0 /)$.
School of ICT, Robotics \& Mechanical Engineering, Hankyong National University, Anseong 17579, Korea; mhkim80@hknu.ac.kr

\begin{abstract}
In this paper, we present the impact of a meander-shaped defected ground structure (MDGS) on the slow-wave characteristics of a lowest-order passband and a low cutoff frequency of the first stopband of an electromagnetic bandgap (EBG) structure for power/ground noise suppression in high-speed integrated circuit packages and printed circuit boards (PCBs). A semi-analytical method is presented to rigorously analyze the MDGS effect. In the analytical method, a closed-form expression for a low cutoff frequency of the MDGS-EBG structure is extracted with an effective characteristic impedance and a slow-wave factor. The proposed analytical method enables the fast analysis of the MDGS-EBG structure so that it can be easily optimized. The analysis of the MDGS effect revealed that the low cutoff frequency increases up to approximately 19\% while comparing weakly and strongly coupled MDGSs. It showed that the miniaturization of the MDGS-EBG structure can be achieved. It was experimentally verified that the low cutoff frequency is reduced from $2.54 \mathrm{GHz}$ to $2.00 \mathrm{GHz}$ by decreasing the MDGS coupling coefficient, which is associated with the miniaturization of the MDGS-EBG structure in high-speed packages and PCBs.
\end{abstract}

Keywords: defected ground structure (DGS); electromagnetic bandgap (EBG); metamaterial; miniaturization; power/ground noise

\section{Introduction}

Power/ground noise, such as simultaneous switching noise (SSN), is a major concern in high-speed circuit designs for achieving high performance and high reliability of electronic systems. As the number of transistors and power consumption of a very large-scale integration (VLSI) increases significantly, power/ground noise severely affects the overall system performance [1-4]. The power/ground noise produces the problem of glitches, timing push-out of digital signals, and power-supply voltage fluctuations. Moreover, it generates noise in power delivery networks (PDNs) of high-speed integrated circuit (IC) packages and printed circuit boards (PCBs). This results in the degradation of the bit error rate performance of high-speed links [5-7], reduction in the on-chip timing margin of clock distribution networks [8-10], and electromagnetic interference (EMI) generated by radiated emission from PDNs [11-14]. The power/ground noise is induced from the resonant cavity modes of the PDNs, which can be considered as a parallel plate waveguide (PPW). Simultaneous switching of a digital circuit excites multiple cavity modes of PDNs, resulting in serious power/ground noise called SSN. In addition, the density of parallel plate modes of PDNs in the high-frequency range substantially increases, such that the parallel plate noise exhibits a wideband spectrum containing multiple high-frequency harmonics. To efficiently mitigate power/ground noise in high-speed IC packages and PCBs, the parallel plate modes of the PDNs must be suppressed over the wideband frequency range.

An effective way of suppressing parallel plate modes in high-speed packages and PCBs is by adopting a PDN wherein an electromagnetic bandgap (EBG) structure is embedded [15-35]. Numerous studies indicate that a PDN employing a metallo-dielectric EBG 
structure successfully suppresses the power/ground noise excited by parallel plate modes with the advantages of simple design, comprehensive examination, rigorous analysis, low fabrication cost, and variety of applications. The metallo-dielectric EBG structure contains a unit cell, wherein a simple resonant component is incorporated using a metal patch and via. The metal patch and via induce capacitance and inductance, resulting in a PPW loaded with an equivalent LC resonator. The periodic arrangement of the LC resonators leads to a decrease in the phase velocity and an increase in the slow-wave factor of the power/ground noise in the PDNs. Moreover, it generates bandgap characteristics (i.e., stopbands) such that the parallel plate modes of the PDNs are suppressed over a wideband frequency range.

EBG structures based on the metallo-dielectric EBG structure have continuously evolved in various digital and microwave applications. The main objective of improving EBG structures is to widen the stopband and reduce the unit cell size of an EBG structure. To achieve this goal, numerous techniques have been presented, namely stacked patches [17-19], multivias [20-22], defected ground structures (DGSs) [23-28], spiral patches [29,30], inductance-enhanced patches [31-33], and high dielectric constant (high k) materials [34,35].

As shown in previous studies, the EBG-based PDN exhibits distinct characteristics in the suppression of parallel plate modes. The mode frequencies of the EBG-based PDN do not continuously appear in the frequency region. The resonant modes of the EBG structure are observed in the frequency range lower or higher than that in the stopband of the EBG structure. Specifically, the lower resonant modes are induced by the inductance and capacitance of the power/ground planes with additional capacitances of the EBG patches and corresponding planes. In the design of EBG-based PDNs, it is preferred that the highest frequency of the lower resonant modes is less than the self-resonance frequency of the decoupling capacitors, such that they are mitigated by simply using decoupling capacitors. The highest frequency of the lower resonant modes is associated with the slow-wave characteristics of the fundamental passband or the low cutoff frequency of the first stopband.

Previous studies mainly focused on improving the first stopband of the metallodielectric EBG structure. They rigorously examined the impact of each technique on the first stopband. Focusing on the enhancement of the first stopband rather than other higher-order stopbands is reasonable and effective because the frequency spectrum of power/ground noise in high-speed digital packages and PCBs consists of harmonics starting from low frequencies. In [20-22], the equivalent circuit and circuit parameters of an EBG unit cell are analytically extracted, and cutoff frequencies are predicted to clearly describe the multivia effect on the fundamental stopband. In [31-33], the techniques of EBG patches etched by various slot patterns are presented to increase the inductance of a shunt LC resonator in an equivalent circuit of an EBG unit cell. This results in the lowering of the fundamental stopband without increasing the size of the EBG unit cell. The effect of etching a patch on the bandwidth of a fundamental stopband is examined using a dispersion analysis based on the analytical model of the EBG unit cell. Several efforts have been made in research to investigate the relationship between the inductance-enhanced patch and first stopband.

One of the evolutionary structures is a metallo-dielectric EBG structure combined with a DGS, as shown in [23-28]. Various shapes of the DGS are employed to extend the bandwidth of the fundamental stopband. Among these DGS patterns, the meandershaped DGS (MDGS) technique has a distinct advantage of increasing the bandwidth of the fundamental stopband with EBG size reduction. In comparison with the other DGS techniques, MDGS substantially lowers the low cutoff frequency, which leads to the miniaturization of the EBG unit cell while still maintaining a wide stopband. In [26,28], the effect of an MDGS with only a single meander segment on a fundamental stopband and size reduction is investigated using an analytical dispersion method. In this analysis, it is assumed that the distance between the strips of a meander segment is very long, ignoring the coupling effect. However, the multiple meander lines with strong-coupling characteristics in the MDGS-EBG structure are inevitably employed to further reduce the 
low cutoff frequency and the size of the EBG unit cell. Therefore, the coupling effect on a fundamental stopband and miniaturization in the MDGS-EBG structure must be analyzed. Furthermore, the impact of MDGSs with loose and tight couplings on the low cutoff frequency needs to be investigated.

In this paper, we focus on examining the MDGS effect on the EBG structure and deriving a new method for the analysis. The coupling effect of the MDGS-EBG structure on the bandwidth of a fundamental passband or a first stopband is thoroughly investigated using a derived dispersion analysis method. In Section 2, the analytical method is derived for the MDGS coupling effect. In Section 3, the impact of MDGS on slow-wave characteristics is analyzed for various cases. In addition, the proposed method and the MDGS effect are verified using full-wave simulation and measurements in Section 4. The paper is concluded with a summary and further works in Section 5.

\section{Analytical Method for Estimation of Low Cutoff Frequency}

In this section, we present an analytical method to explore the effect of MDGS on the slow-wave characteristics and low cutoff frequency of the EBG structure. The proposed method is a semi-empirical method based on full-wave simulation, scattering parameter (S-parameter) extraction method, and Floquet theory. A dispersion diagram for the fundamental passband and low cutoff frequency of the first stopband is derived using an analytical method. A unit cell of the MDGS-EBG structure in Figure 1 is employed as an example of the application of the proposed method. The unit cell of the MDGS-EBG structure consists of three conductors: a power plane, patch, and defected ground plane. A defected ground plane is connected to a patch using a via. The defected ground plane is perforated using a meander pattern, which results in two meander lines comprising of multiple meander segments. The meander segment is two parallel-coupled lines. They are equal in length and connected at one end. The connection length of the end point should be non-zero in this study, although many previous studies on coupled lines ignore the length for simplicity of analysis. The number of meander segments in a meander line is represented as $\mathrm{N}$. Three meander segments $(\mathrm{N}=3)$ are depicted in Figure 1; however, the proposed method is not limited to this number.

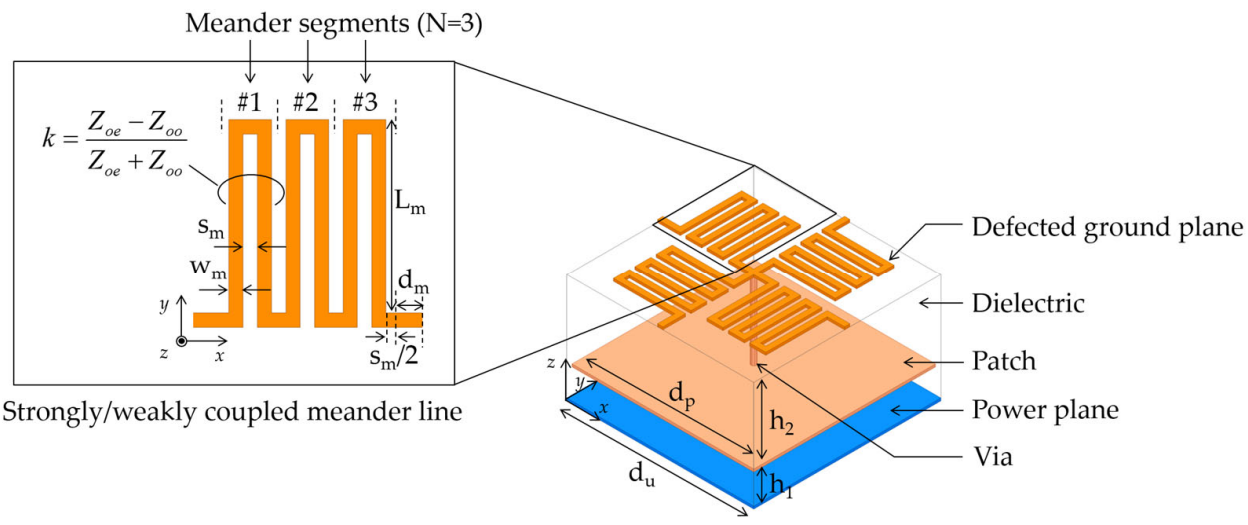

Figure 1. Multi-meander segments in a unit cell of MDGS-EBG structure and design parameters.

In this study, we employ a coupling coefficient $k$ to indicate an EBG unit cell or a meander line in it. Because it is difficult to derive a coupling coefficient for a multi-coupled meander line, $\mathrm{k}$ is defined for a single meander segment, which is the ratio of the difference of even- and odd-mode characteristic impedances of coupled lines in a single meander segment to their sum. The coupling coefficient $\mathrm{k}$ is strongly related to the geometrical parameters of the width $\left(w_{m}\right)$ of a meander line and the spacing $\left(s_{m}\right)$ between adjacent strips of parallel-coupled lines. It is calculated using an empirical equation or an electromagnetic simulation. The other geometrical variables of the MDGS-EBG structure are unit cell length 
$\left(d_{u}\right)$, patch length $\left(d_{p}\right)$, via radius $\left(r_{v}\right)$, dielectric thickness between power plane and patch $\left(h_{1}\right)$, and dielectric thickness between defected ground plane and patch $\left(h_{2}\right)$.

For simplicity, coupling between a meander segment and the other meander segments is not considered while determining the coupling coefficient $\mathrm{k}$. However, the main characteristics of the MDGS-EBG structure originate from the multi-coupling between the meander segments. The propagation characteristics of the meander line are affected by couplings between adjacent strips in a coupled line and nearby meander segments. Owing to the complicated coupling, it is difficult to obtain an analytical solution for coupling of the meander line in terms of geometrical parameters [36-41]. Hence, the characteristics of the meander line are typically analyzed using full-wave simulation.

The impact of MDGS on the slow-wave characteristics of the fundamental passband and a low cutoff frequency of the first stopband can be examined using dispersion analysis based on the full-wave simulation of the entire unit cell of the MDGS-EBG structure. However, the method using full-wave simulation for the entire unit cell is time-consuming. The boundary configuration of the MDGS-EBG structure is dissimilar to the other EBG structure evolved from the metallo-dielectric EBG structure. Owing to the MDGS, an air box is placed above the defected ground plane, and a perfectly matched layer or radiation boundary is adopted. This results in an extremely large computation time during the parametric sweeping of the full-wave simulation. Thus, it does not provide an insight into the MDGS-EBG design. However, the complex coupling of the meander line should be captured because it mainly affects the dispersion characteristics of the MDGSEBG structure. Considering the extreme difficulty in extracting an analytical solution for dispersion characteristics for strongly and weakly coupled meander lines, a new method for dispersion analysis of the MDGS-EBG structure is proposed, as depicted in Figure 2.



Figure 2. Proposed semi-analytical method for MDGS effect on slow-wave characteristics and low cutoff frequency of EBG structure.

The proposed method combines the full-wave simulation and analytical equation to achieve a reasonable computation time and accuracy. The results of the semi-empirical method are a dispersion equation and a closed-form expression for the slow-wave characteristics of the fundamental passband and a low cutoff frequency of the first stopband, respectively. Because the MDGS-EBG structure is periodic, Floquet analysis based on the unit cell is adopted. The proposed semi-analytical method consists of four main steps, as illustrated in Figure 2. In the brief explanation of the procedure, the coupling effect of the MDGS pattern is captured using full-wave simulation of a part of the unit cell. From the result, an effective characteristic impedance and slow-wave factor are extracted using the S-parameter extraction method, which are important parameters for the analysis of the MDGS effect. The unit cell of the MDGS-EBG structure was modeled as an equivalent circuit. Applying the Floquet theorem to the circuit, an analytical solution for a dispersion diagram is derived. A closed-form expression for a low cutoff frequency is further extracted with small-angle approximation. 
Specifically, in the first step of the proposed semi-analytical method, we perform fullwave simulation of half of the unit cell removing the patch and via, namely a meander line with a corresponding reference plane. This approach achieves an effective analysis owing to the simulation region reduction. The computation time can be substantially reduced such that we can obtain enormous simulation results, sweeping design variables related to coupling effects. In this step, we obtain the S-parameters of the strongly and weakly coupled meander lines.

Next, an effective characteristic impedance $\left(Z_{0 m}\right)$, propagation constant $\left(\gamma_{m}\right)$, and slow-wave factor $\left(\rho_{S W F}\right)$ are extracted using the S-parameter extraction method in [42]. $Z_{0 m}$, $\gamma_{m}$, and $\rho_{\mathrm{SWF}}$ are the major parameters that are used in an equivalent circuit model of the unit cell of the MDGS-EBG structure. As shown in [42], $Z_{0 m}$ is extracted as follows:

$$
Z_{0 m}=Z_{0} \cdot \sqrt{\frac{\left(1+S_{11, m}\right)^{2}-S_{21, m}^{2}}{\left(1-S_{11, m}\right)^{2}-S_{21, m}^{2}}}
$$

where $S_{i j, m}(i=1,2, j=1,2)$ is a reflection or transmission component of the S-parameter of the meander line. $Z_{0}$ is the reference impedance of $50 \Omega$.

The propagation constant $\left(\gamma_{\mathrm{m}}\right)$ and slow-wave factor $\left(\rho_{S W F}\right)$ are given by

$$
\begin{gathered}
\gamma_{m}=\frac{2}{d_{u}} \ln \left\{\frac{1-S_{11, m}^{2}+S_{21, m}^{2}}{2 S_{21, m}}+\left(\frac{\left(S_{11, m}^{2}-S_{21, m}^{2}+1\right)^{2}-\left(2 S_{11, m}\right)^{2}}{\left(2 S_{21, m}\right)^{2}}\right)^{1 / 2}\right\}, \\
\rho_{S W F}=\frac{\operatorname{Im}\left(\gamma_{m}\right)}{\beta_{o}}=\frac{\beta_{m}}{\beta_{o}},
\end{gathered}
$$

where $\beta_{o}$ is the phase constant of light in vacuum.

To completely extract the propagation characteristics of the unit cell of the MDGS-EBG structure, an equivalent circuit model is illustrated in Figure 3. It comprises two equivalent transmission lines and an $\mathrm{LC}$ resonator. The equivalent transmission lines have $Z_{0 m}, \beta_{m}$, and $\rho_{S W F}$ derived from the previous steps. Its length is half of $d u$. The via connecting a defected ground plane and a patch leads to the inductance $\left(L_{v}\right)$ of the LC resonator. The capacitance $\left(C_{p}\right)$ is induced by a patch and a corresponding power plane. $L_{v}$ and $C_{p}$ are calculated using analytical equations as follows [43].

$$
\begin{gathered}
L_{v}=\frac{\mu_{0} h_{2}}{4 \pi}\left(2 \ln \left(\frac{d_{u}}{r_{v} \sqrt{\pi}}\right)+\left(\frac{r_{v} \sqrt{\pi}}{d_{u}}\right)^{2}-1\right), \\
C_{p}=\varepsilon_{o} \varepsilon_{r} \frac{d_{p}^{2}}{h_{1}} .
\end{gathered}
$$

To derive its effective phase constant, the unit cell of the MDGS-EBG structure is modeled as the equivalent transmission line, of which an effective phase constant and a length are $\beta_{\text {eff }}$ and $d_{u}$, respectively. The ABCD matrix for the unit cell of the MDGS-EBG structure is equal to the product of the $\mathrm{ABCD}$ matrices of the equivalent transmission lines and the LC resonator in shunt with them as follows:

$$
\left(\begin{array}{cc}
A_{\text {eff }} & B_{\text {eff }} \\
C_{\text {eff }} & D_{\text {eff }}
\end{array}\right)=\left(\begin{array}{cc}
\cos \left(\frac{\beta_{m} d_{u}}{2}\right) & j Z_{0 m} \sin \left(\frac{\beta_{m} d_{u}}{2}\right) \\
j Z_{0 m}^{-1} \sin \left(\frac{\beta_{m} d_{u}}{2}\right) & \cos \left(\frac{\beta_{m} d_{u}}{2}\right)
\end{array}\right) \times\left(\begin{array}{cc}
1 & 0 \\
\frac{j \omega C_{p}}{1-\omega^{2} L_{v} C_{p}} & 1
\end{array}\right)\left(\begin{array}{cc}
\cos \left(\frac{\beta_{m} d_{u}}{2}\right) & j Z_{0 m} \sin \left(\frac{\beta_{m} d_{u}}{2}\right) \\
j Z_{0 m}^{-1} \sin \left(\frac{\beta_{m} d_{u}}{2}\right) & \cos \left(\frac{\beta_{m} d_{u}}{2}\right)
\end{array}\right)
$$




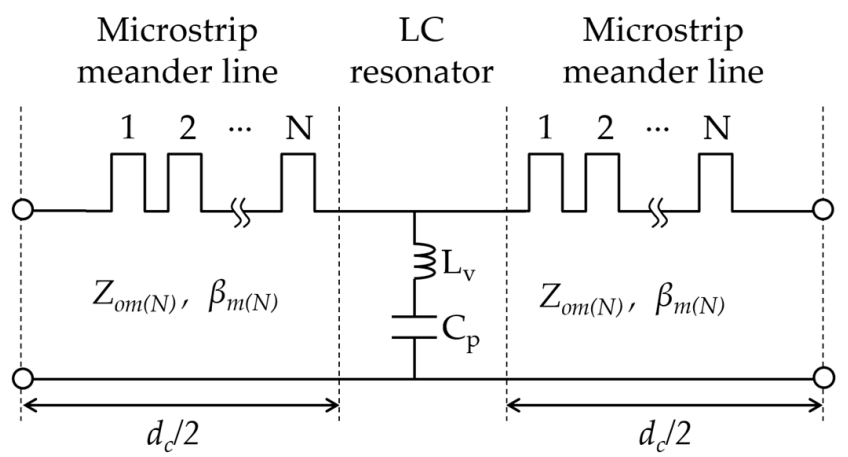

Figure 3. Equivalent circuit model based on effective characteristic impedance and propagation constant for dispersion analysis.

The evaluation of $\beta$ eff from the above equation yields the analytical equation for the effective phase constant of the MDGS-EBG structure as follows:

$$
\cos \left(\beta_{e f f} d_{u}\right)=\cos \left(\beta_{m} d_{u}\right)-\frac{\omega C_{p} Z_{0 m}}{2\left(1-\omega^{2} L_{v} C_{p}\right)} \sin \left(\beta_{m} d_{u}\right) .
$$

The numerical analysis of (7) provides the dispersion characteristics of the fundamental passband and first stopband. It can demonstrate a low cutoff frequency. However, for a more convenient assessment of the MDGS effect on a low cutoff frequency, an explicit expression for a low cutoff frequency is extracted by applying the condition of $\beta_{\text {eff }} d_{u}=\pi$, which results in the left-hand side of (7) being equal to -1 . For further simplification of (7), we can inspect the $\beta_{m} d_{u}$ parameter. It is noted that $\beta \mathrm{m}$ is the phase constant of the meander line acquired from the S-parameter extraction method. The $\beta_{m} d_{u}$ at a low cutoff frequency is given by

$$
\beta_{m} d_{u}=\rho_{S W F} \beta_{o} d_{u}=\frac{2 \pi f_{L} \cdot \rho_{S W F} \cdot d_{u}}{c},
$$

where $f_{L}$ is the low cutoff frequency; $c$ is the speed of light, which is equal to $3 \times 10^{8} \mathrm{~m} / \mathrm{s}$. Considering the typical values of $\rho_{S W F}, d_{u}$, and $f_{L}$ of the MDGS-EBG structure in high-speed packages and PCBs, they are extremely small in comparison with c. Consequently, we can assume that $\cos \left(\beta_{m} d_{u}\right)$ and $\sin \left(\beta_{m} d_{u}\right)$ are approximately equal to 1 and $\beta_{m} d_{u}$, respectively, in the right-hand side of (7).

With the aforementioned assumptions, a closed-form expression for the low cutoff frequency is derived as follows:

$$
f_{L}=\frac{1}{2 \pi \sqrt{C_{p}\left(L_{v}+L_{e f f}\right)}},
$$

where

$$
L_{e f f}=\frac{\rho_{S W F} Z_{0 m} d_{u}}{4 c} .
$$

The low cutoff frequency of the MDGS-EBG structure can be simply expressed as the resonance frequency of the effective $\mathrm{LC}$ resonator, where the capacitance value is $C_{p}$ and the inductance value is effectively determined as the product of $\rho_{S W F}, Z_{0 m}$, and $d_{u}$. As can be seen in (9) and (10), adjustment of a low cutoff frequency is achieved by varying a slow-wave factor, an effective characteristic impedance of the meander line, and a unit cell size, whereas the $L_{\text {eff }}$ of the previous metallo-dielectric EBG structure is mainly affected by the unit cell size. In the previous metallo-dielectric EBG structure, the unit cell size needed to be increased to lower the low cutoff frequency, but a low cutoff frequency of the MDGS-EBG structure can decrease without increasing the unit cell size. In other words, the unit cell of the MDGS-EBG structure is smaller than that of the previous EBG structure 
when these EBG structures have the same low cutoff frequency. This is the distinguishing feature anticipated by the MDGS effect. Using the semi-analytical method presented herein, we can examine the effect of the MDGS on the slow-wave characteristics of the fundamental passband and the low cutoff frequency of the first stopband for the MDGS-EBG structure containing multiple meander segments.

\section{Results and Discussions}

In this section, the MDGS effect is analyzed using the proposed semi-analytical method. Dispersion characteristics of the fundamental passband and a low cutoff frequency of the first stopband are examined with various coupling coefficients and numbers of meander segments.

\subsection{MDGS Effect on $Z_{o m}, \rho_{S W F}$, and $f_{L}$}

To explore the MDGS effect, we employ six exemplary MDGS-EBG structures, including the dimensions of the design parameters, as presented in Table 1 . The values of the design parameters are chosen considering their practical use in high-speed packages and PCBs. The MDGS-EBG structure contains three meander segments $(\mathrm{N}=3)$ in half of a unit cell, as can be observed in Figure 1. For further inspection of the coupling effect, the spacing $\left(s_{m}\right)$ between adjacent strips in the meander segment changes from $0.05 \mathrm{~mm}$ to $0.30 \mathrm{~mm}$ by a step of $0.05 \mathrm{~mm}$, while the total length of the meander line $\left(L_{t}\right)$ remains constant in all cases. The corresponding coupling coefficients $k$ are $0.54,0.44,0.38,0.32$, 0.28 , and 0.25 . As previously described, the value of $k$ is calculated using a single meander segment with perfect magnetic walls at ports and is used to simply indicate a specific meander segment. The values of $k$ for various dimensions are shown in Figure $4 \mathrm{a}$. Both $k$ and $\mathrm{N}$ can represent a complete meander line. By varying the value of $k$, we can investigate the impact of a strongly and weakly coupled MDGS on the dispersion characteristics and a low cutoff frequency.

Table 1. Design Parameters and Physical Dimensions.

\begin{tabular}{|c|c|c|c|c|c|}
\hline \multirow{2}{*}{ TVs ${ }^{1,2,3}$} & \multicolumn{5}{|c|}{ Parameters } \\
\hline & $s_{m}(\mathrm{~mm})$ & $d_{m}(\mathrm{~mm})$ & $k$ & $Z_{0 m}(\Omega)$ & $\rho_{S W F}$ \\
\hline 1 & 0.05 & 0.80 & 0.54 & 81.7 & 4.93 \\
\hline 2 & 0.10 & 0.65 & 0.44 & 93.2 & 5.23 \\
\hline 3 & 0.15 & 0.50 & 0.38 & 98.9 & 5.49 \\
\hline 4 & 0.20 & 0.35 & 0.32 & 102.6 & 5.72 \\
\hline 5 & 0.25 & 0.20 & 0.28 & 105.0 & 5.94 \\
\hline 6 & 0.30 & 0.05 & 0.25 & 106.8 & 6.11 \\
\hline
\end{tabular}

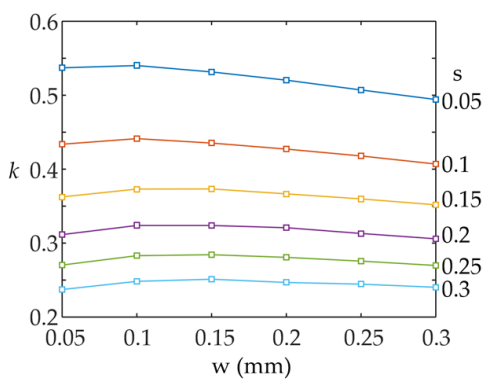

(a)

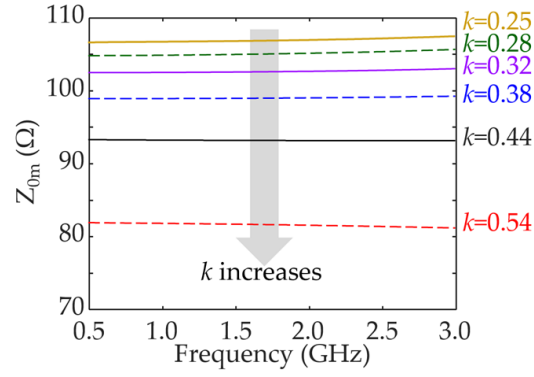

(b)

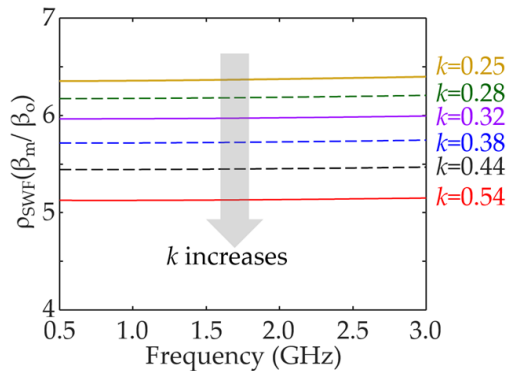

(c)

Figure 4. (a) coupling coefficient $\mathrm{k}$ with various dimensions; (b) Effective characteristic impedance; (c) slow-wave factor for MDGSs with various coupling coefficients. 
Applying the proposed semi-analytical method to the meander lines of the six test vehicles (TVs), the S-parameters of the meander lines containing three meander segments are obtained using the full-wave simulation. $Z_{0 m}$ and $\rho_{S W F}$ are extracted from (1) to (3), as depicted in Figure 4. All $Z_{0 m}$ exhibit quasi-static characteristics in the frequency range of interest such that we can use a single value of $Z_{0 m}$ in the wideband frequency range. The values of $Z_{0 m}$ for $k=0.54,0.44,0.38,0.32,0.28$, and 0.25 are equal to $81.7,93.2,98.9$, 102.6, 105.0, and $106.8 \Omega$, respectively. It is observed in Figure $4 \mathrm{~b}, \mathrm{c}$ that $Z_{0 m}$ decreases as $k$ increases. It is noted that a strongly coupled meander line has a lower $Z_{0 m}$ than a weakly coupled meander line. We find a similar result for $\rho_{S W F}$. The values of $\rho_{S W F}$ with $k=0.54,0.44,0.38,0.32,0.28$, and 0.25 are equal to $4.93,5.23,5.49,5.72,5.94$, and 6.11 , respectively. This implies that the slow-wave effect of the meander line decreases when the coupling coefficient increases. For meander lines having the same total length, the propagation velocity of a weakly coupled line is slower in comparison with that of a strongly coupled line.

Using the aforementioned $Z_{0 m}$ and $\rho_{S W F}$ values, the slow-wave characteristics of the MDGS-EBG structures are analyzed. In Figure 5a, the dispersion diagrams of six TVs are acquired numerically by calculating the dispersion equation of (7) with the extracted $Z_{0 m}$ and $\rho_{S W F}$ values. As anticipated, all TVs exhibit slow-wave characteristics in comparison with the light line. However, the MDGS effect on slow-wave characteristics is different in accordance with the coupling coefficient $k$. It is observed that the MDGS effect is mitigated as $k$ increases. With the same length of the meander line and the same size of the unit cell, the impact of MDGS on the slow-wave characteristics changes according to the coupling coefficient $k$. It was found that we can adjust the slow-wave effect of the MDGS-EBG structure using the coupling coefficient, thus controlling a low cutoff frequency without varying the size or thickness of the unit cell size. The low cutoff frequencies for $k$ of 0.54 , $0.44,0.38,0.32,0.28$, and 0.25 are obtained as $2.54,2.31,2.19,2.10,2.05$, and $2.00 \mathrm{GHz}$, respectively, as depicted in Figure 5a. It was found that the low cutoff frequency reduces as $k$ decreases. This result can be anticipated from (9). The equation, extracted herein, indicates that the low cutoff frequency is inversely proportional to the root of $Z_{0 m}$ and $\rho_{S W F}$. It is already seen in Figure 4 that the $Z_{0 m}$ and $\rho_{S W F}$ reduce when $k$ increases. Consequently, a high $k$ leads to a high cutoff frequency. The cutoff frequencies of the MDGS-EBG structure with the weakly $(k=0.25)$ and strongly $(k=0.54)$ coupled lines are 2.00 and $2.54 \mathrm{GHz}$, respectively. The variation of the low cutoff frequency is $27 \%$. Remarkably, we can achieve a substantial change in the low cutoff frequency of the MDGS-EBG structure by adjusting the coupling coefficient $k$. Following these important findings, we can emphasize the significance of the proposed analysis method for the MDGS effect.

To verify the MDGS effect and the proposed method, full-wave simulations for the same TVs of the MDGS-EBG structures are performed. The dispersion diagrams in Figure $5 b$ are obtained using the Floquet theorem with the full-wave simulation result for the unit cell of the MDGS-EBG structure. It is also observed in the full-wave simulation result that the MDGS coupling significantly affects the low cutoff frequency of the first stopband, which is the same result as obtained in the proposed semi-analytical method. The low cutoff frequencies for $k$ of $0.25,0.28,0.32,0.38,0.44$, and 0.54 are equal to 1.79 , $1.81,1.83,1.86,1.96$, and $2.13 \mathrm{GHz}$, respectively. It is also demonstrated that the low cutoff frequency significantly increases up to $19 \%$ as the coupling coefficient changes from 0.25 to 0.54 through the full-wave simulation. In addition, the proposed analytical method achieves a fast analysis. The computation time of the analytical method is $240 \mathrm{~s}$ while that of the full-wave simulation is $3332 \mathrm{~s}$. The proposed analytical method substantially reduces the computation time by up to $92.8 \%$.

The proposed method provides an additional method for simple estimation of a low cutoff frequency, as derived in (9). In Figure 6, the low cutoff frequencies extracted from the closed-form expression of (9) are shown in combination with the results of the dispersion Equation (7) and full-wave simulation. The low cutoff frequencies of (9) for $k$ of $0.25,0.28$, $0.32,0.38,0.44$, and 0.54 are $2.0,2.05,2.1,2.19,2.31$, and $2.54 \mathrm{GHz}$, respectively. It is depicted 
in Figure 6 that the proposed method of (7) and (9) correlates well with the full-wave simulation although their values show a difference. This discrepancy originates from the assumption that $L_{v}$ is extracted without considering the coupling effect, and the small-angle approximation of a phase constant of a meander line is considered.

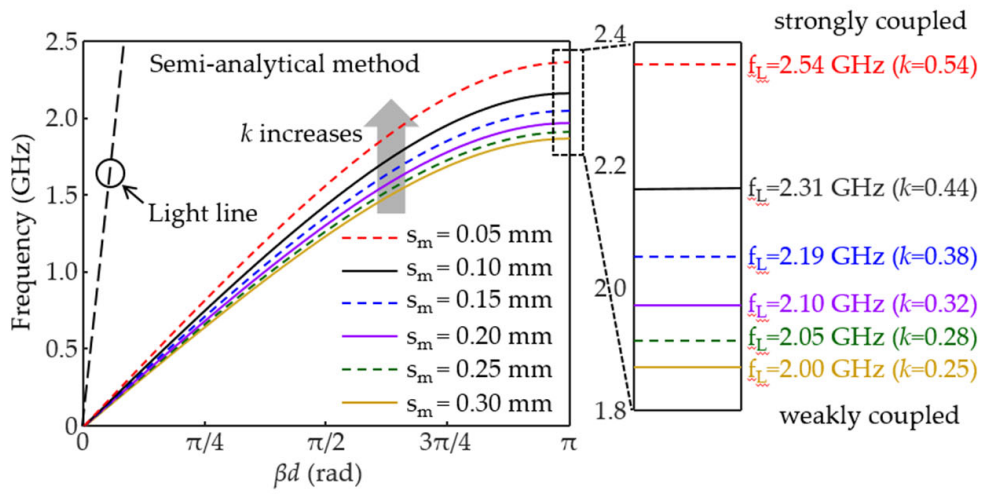

(a)

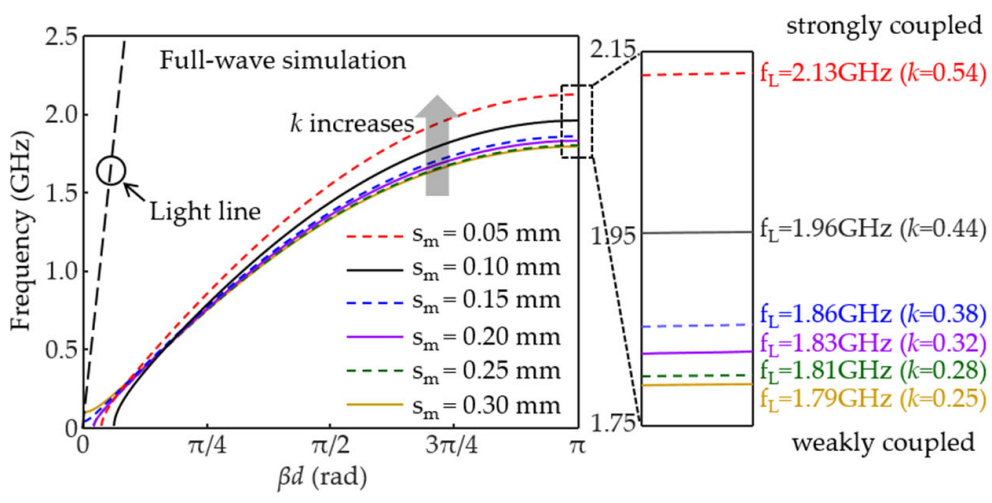

(b)

Figure 5. Dispersion diagrams of (a) proposed semi-analytical method; (b) full-wave simulation for MDGS effect analysis.



Figure 6. Analysis of MDGS effect on low cutoff frequency using the proposed method and fullwave simulation.

\subsection{Reduction in $N$ Using MDGS Effect}

To comprehensively verify the proposed method and the MDGS effect, further exploration of the low cutoff frequencies for various MDGS-EBG structures is performed. The 
MDSG-EBG structures with unit cell sizes of 6 and $7 \mathrm{~mm}$ and a meander segment number of 2 are additionally analyzed. In Figures 7 and 8, we compare the low cutoff frequencies of (9) and a full-wave simulation for the MDGS-EBG structure with N values of 2 and $3, k$ of $0.54,0.44,0.38,0.32,0.28$, and 0.25 , du of 5, 6, and $7 \mathrm{~mm}$. Figure 7 shows that the low cutoff frequency decreases as the coupling coefficient decreases. The coupling of MDGS substantially affects the low cutoff frequency. For instance, the variation between the low cutoff frequencies of the weakly and strongly coupled structures with $\mathrm{N}=2$ and $d_{u}=5 \mathrm{~mm}$ is approximately $16.8 \%$ in Figure 7 a.

In Figure 7a-f, the MDGS effect is consistently shown for all TVs. It is confirmed that the proposed method successfully captures the MDGS effect in comparison with the full-wave simulation result, although a discrepancy between the proposed method and the full-wave simulation is observed. The tendency of the MDGS effect of the proposed method shows a good correlation with that of the full-wave simulation. As mentioned earlier, the error results from the assumption of ignoring the coupling effect while extracting $L_{v}$ and approximating the phase constant. Comparing the results $(\mathrm{N}=2)$ in Figure 7 with the results $(\mathrm{N}=3)$ in Figure 8 , we can observe that the error increases as the coupling effect further increases. In Figure 8, we investigate the differences in the low cutoff frequencies of strongly $(k=0.54)$ and weakly $(k=0.25)$ coupled MDGS-EBG structures to emphasize the MDGS effect. Figure $8 \mathrm{a}$,b summarize $f_{L}(k=0.54)-f_{L}(k=0.25)$ for the MDGS-EBG structures with $\mathrm{N}$ values of 2 and 3, respectively. For all TVs, it is observed that the MDGS coupling affects the low cutoff frequency, and its effect is significant. Lowering the coupling coefficient of the MDGS leads to a reduction in the low cutoff frequency without increasing the unit cell size or dielectric thickness.

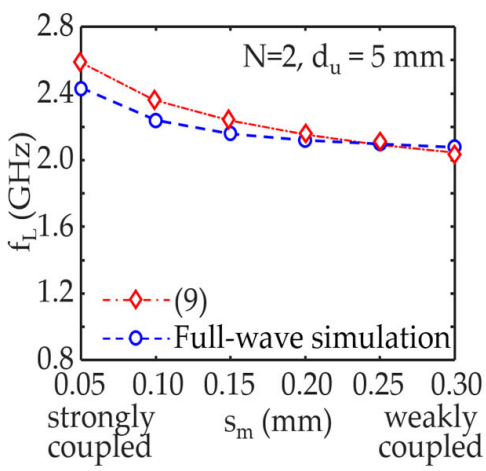

(a)

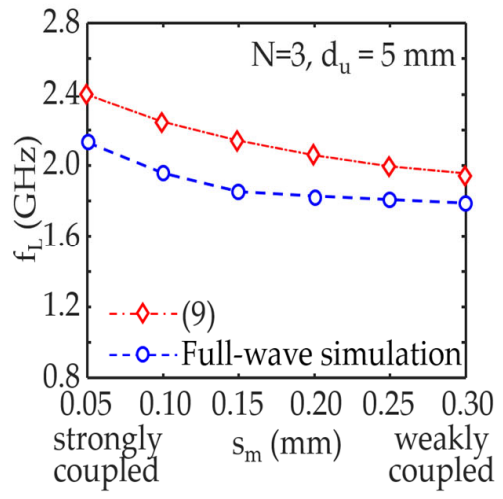

(d)

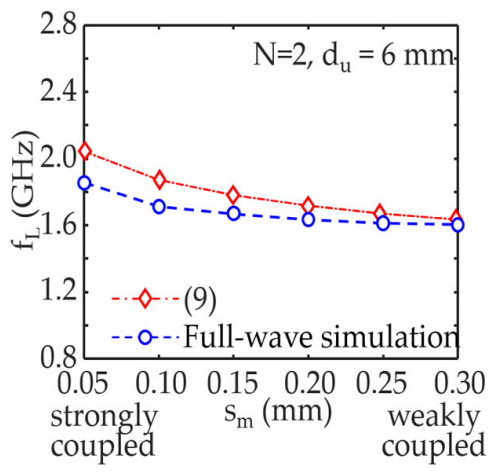

(b)

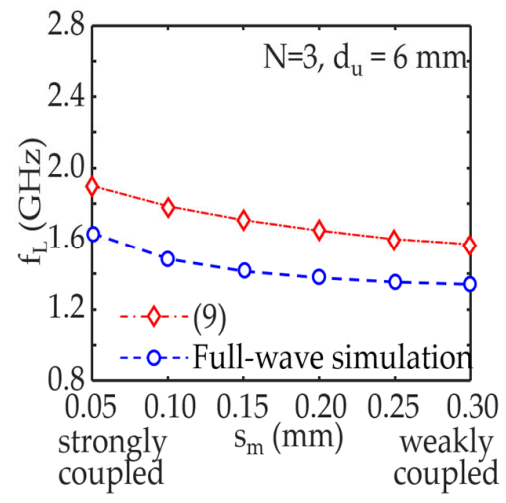

(e)

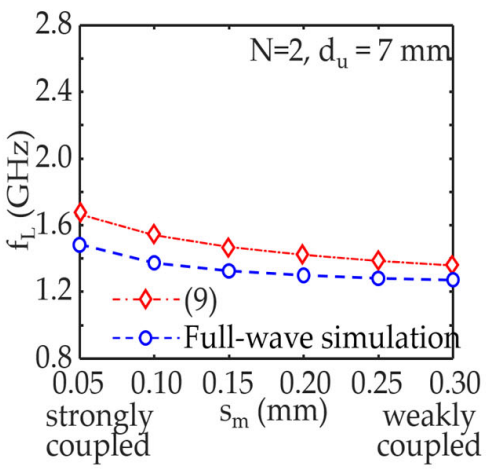

(c)

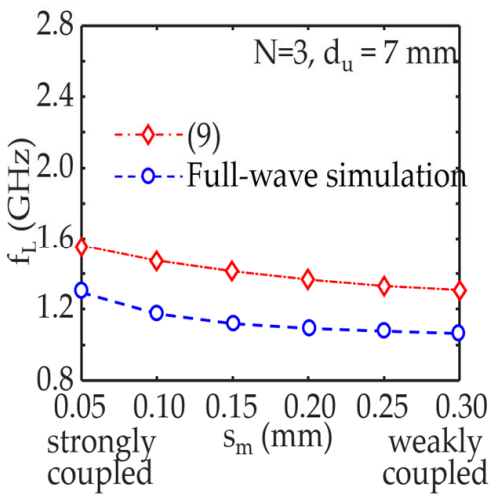

$(\mathbf{f})$

Figure 7. Comparison of MDGS effect on low cutoff frequency of the MDGS-EBG structures with (a) $\mathrm{N}=2, d_{u}=5 \mathrm{~mm}$; (b) $\mathrm{N}=2, d_{u}=6 \mathrm{~mm}$; (c) $\mathrm{N}=2, d_{u}=7 \mathrm{~mm} ;(\mathbf{d}) \mathrm{N}=3, d_{u}=5 \mathrm{~mm}$; (e) $\mathrm{N}=3$, $d_{u}=5 \mathrm{~mm} ;(\mathbf{f}) \mathrm{N}=3, d_{u}=5 \mathrm{~mm}$. 


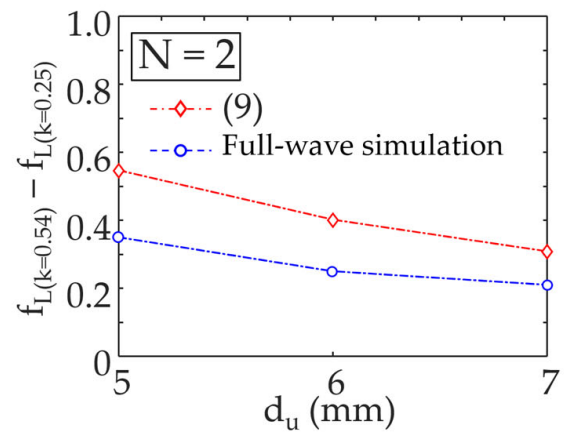

(a)

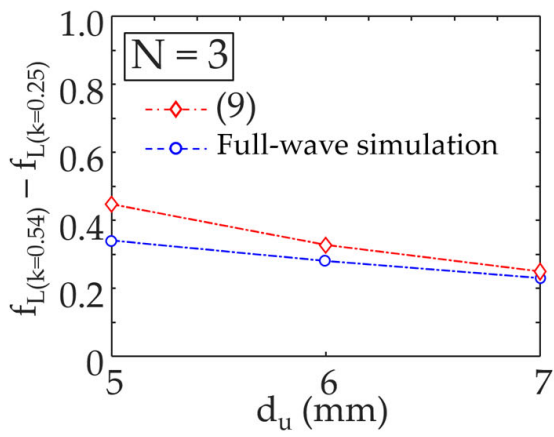

(b)

Figure 8. Differences in low cutoff frequencies between the MDGS-EBG structures with strongly and weakly coupled MDGS with (a) $\mathrm{N}=2 ;$ (b) $\mathrm{N}=3$.

Furthermore, the significance of the MDGS effect is demonstrated by comparing a strongly coupled MDGS-EBG structure with $\mathrm{N}+1$ and a weakly coupled one with $\mathrm{N}$, which are the MDGS-EBG structures including the same dimensions of design parameters except the number of meander segments. As depicted in Figure 9, the low cutoff frequency of the MDGS-EBG structure with $(\mathrm{N}=3, k=0.54)$ is compared with that with $(\mathrm{N}=2, k=0.25)$ for various unit cell sizes. The total length of the meander line of $\mathrm{N}=3$ is longer than that of $\mathrm{N}=2$. However, the low cutoff frequency of $\mathrm{N}=2$ is lower than that of $\mathrm{N}=3$. The same result can be obtained in the comparison of $(\mathrm{N}=4, k=0.54)$ and $(\mathrm{N}=3, k=0.25)$ in Figure 10b. The amount of reduction in the low cutoff frequency even increases for this case. This finding is an original feature of the MDGS-EBG structure. The MDGS-EBG structure has the advantages of miniaturization and wideband stopband. However, IR drop induced from the meander section is problematic. The results proposed in this paper provide a way to reduce the IR drop problem with maintaining the advantages of the MDGS-EBG structure.


Figure 9. Unique characteristics of $f_{L}$ reduction in comparison of MDGS-EBG structures with $\mathrm{N}$ and $\mathrm{N}+1$.



(a)



(b)

Figure 10. Fabricated test vehicles of (a) TV A $(k=0.36)$; (b) TV B $(k=0.09)$. 
The MDGS-EBG structure and the proposed analytical method are compared with previous studies as shown in Table 2. The unit cell size of the MDGS-EBG structure can be reduced with adjusting $k$, thus miniaturizing the MDGS-EBG structure. The $k$ effect on the EBG structure is analyzed herein. The proposed analytical method shows the high flexibility and short computation time for the analysis, whereas the previous studies do not satisfy both.

Table 2. Performance comparison.

\begin{tabular}{cccccc}
\hline \multirow{2}{*}{ Ref. } & \multirow{2}{*}{$f_{L}(\mathbf{G H z})$} & $\begin{array}{c}\text { Unit Cell } \\
\text { Length }(\mathbf{m m})\end{array}$ & $\begin{array}{c}\text { k Effect } \\
\text { Analysis }\end{array}$ & & \multicolumn{2}{c}{ Analysis Method } \\
\cline { 5 - 6 } & 2.91 & 6.6 & N.A. & High & Too long \\
\hline 15$]$ & 0.68 & 20 & N.A. & Low & Short \\
{$[20]$} & 1.6 & 8 & N.A. & High & Too long \\
{$[23]$} & 0.7 & 12.2 & No & Low & Short \\
{$[26]$} & 5 & Yes & High & Short \\
This work & 1.79 & 5 & & &
\end{tabular}

\section{Experimental Validation}

To experimentally verify the MDGS effect on reduction at a low cutoff frequency, two TVs of the strongly and weakly coupled MDGS-EBG structures were fabricated using a conventional PCB fabrication, as shown in Figure 10. The dimensions of the design parameters are presented in Table 3. TVs A and B are the MDGS-EBG structure with strongly $(k=0.36)$ and weakly $(k=0.09)$ coupled lines with $\mathrm{N}=1$, respectively. The TVs contain a $7 \times 7$ EBG array. The ports are located at the edge of the EBG array. The Sparameters were measured using a vector network analyzer and 500- $\mu \mathrm{m}$-pitch-GS probes.

Table 3. Design Parameters and Physical Dimensions.

\begin{tabular}{ccc}
\hline Parameters & TV A & TV B \\
\hline$k$ & 0.36 & 0.09 \\
$s_{m}$ & 0.3 & 1.4 \\
$d_{m}$ & 1.2 & 0.7 \\
$w_{m}$ & 0.2 & 0.2 \\
$L_{m}$ & 2.6 & 2.6 \\
$d_{u}$ & 6.2 & 6.2 \\
$d_{p}$ & 6.0 & 6.0 \\
$h_{1}$ & 0.1 & 0.1 \\
$h_{2}$ & 0.8 & 0.8 \\
$L_{t}$ & 11.4 & 11.4 \\
$\mathrm{~N}$ & 1 & 1 \\
$Z_{0 m}$ & 88.1 & 110.5 \\
$\rho_{S W F}$ & 3.63 & 3.78 \\
\hline
\end{tabular}

Figure 11 depicts the measured $S_{21}$ parameters of TVs A and B. The measured low cutoff frequencies with $\left|\mathrm{S}_{21}\right|$ of $-40 \mathrm{~dB}$ of TVs $\mathrm{A}(k=0.36)$ and $\mathrm{B}(k=0.09)$ are $1.21 \mathrm{GHz}$ and $1.14 \mathrm{GHz}$, respectively. The low cutoff frequency increases up to $6.1 \%$ when the MDGS coupling coefficient changes from 0.09 to 0.36 . Even if the fabricated TVs include the meander section of only $\mathrm{N}=1$, it is observed that the low cutoff frequency is significantly affected. Consequently, it is expected that the MDGS effect becomes significant and the low cutoff frequency substantially reduces as the number of meander sections $(\mathrm{N})$ increases which results in the IR drop reduction and the opportunity of miniaturizing EBG structures. 


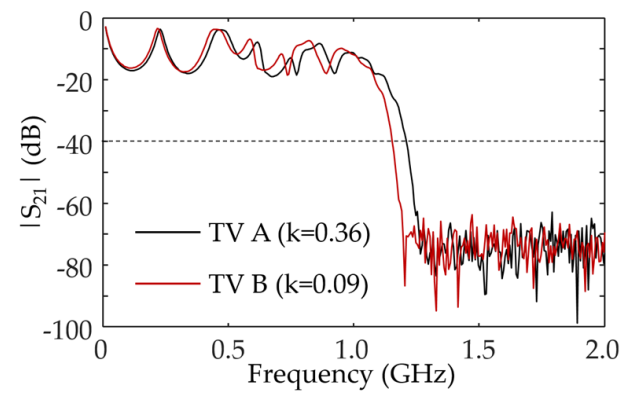

Figure 11. Measurements of strongly and weakly coupled MDGS-EBG structures for verification of MDGS effect.

\section{Conclusions}

We presented the MDGS effect on the slow-wave characteristics and low cutoff frequency of the EBG structure in high-speed IC packages and PCBs. The analysis combining the full-wave simulation with the derived equations was extracted and verified. Using the proposed method, a rigorous analysis of the MDGS effect was performed. The proposed method achieved a fast analysis of the MDGS-EBG structure. It was validated using full-wave simulations and measurements. The analysis of the MDGS effect reflects that the slow-wave factor and low cutoff frequency were significantly affected by the coupling coefficient of the meander segment. For the demonstration case, the $f_{L}$ change was up to $19 \%$ when the coupling coefficient was varied. Furthermore, the low cutoff frequency of the weakly coupled MDGS-EBG structure with the meander segment number of $\mathrm{N}$ was lower than that of the strongly coupled MDGS-EBG structure with N+1. According to the results, we can conclude that the total length of the MDGS is reduced without increasing the low cutoff frequency which results in miniaturizing the EBG structure and solving an IR drop problem of a long meander line of the MDGS.

Funding: This work was supported by the National Research Foundation of Korea (NRF) grant funded by the Korea government (Ministry of Science and ICT) (NRF-2019R1C1C1005777).

Conflicts of Interest: The author declares no conflict of interest.

\section{References}

1. Wu, T.-L.; Chuang, H.-H.; Wang, T.-K. Overview of power integrity solutions on package and PCB: Decoupling and EBG isolation. IEEE Trans. Electromagn. Compat. 2010, 52, 346-356. [CrossRef]

2. McCredie, B.D.; Becker, W.D. Modeling, measurement, and simulation of simultaneous switching noise. IEEE Trans. Compon. Packag. Manuf. Technol. Part B. 1996, 19, 461-472. [CrossRef]

3. Tang, K.T.; Friedman, E.G. Simultaneous switching noise in on-chip CMOS power distribution networks. IEEE Trans. Very Large Scale Integr. (VLSI) Syst. 2002, 10, 487-493. [CrossRef]

4. Shim, Y.; Park, J.; Kim, J.; Song, E.; Yoo, J.; Pak, J.; Kim, J. Modeling and Analysis of Simultaneous Switching Noise Coupling for a CMOS Negative-Feedback Operational Amplifier in System-in-Package. IEEE Trans. Electromagn. Compat. 2009, 51, 763-773. [CrossRef]

5. Heydari, P. Analysis of the PLL jitter due to power/ground and substrate noise. IEEE Trans. Circuits Syst. I Regul. Pap. 2004, 51, 2404-2416. [CrossRef]

6. Sui, C.; Ren, L.; Gao, X.; Pan, J.; Drewniak, J.L.; Beetner, D.G. Predicting Statistical Characteristics of Jitter Due to Simultaneous Switching Noise. IEEE Trans. Electromagn. Compat. 2016, 58, 249-256. [CrossRef]

7. Souilem, M.; Tripathi, J.N.; Dghais, W.; Belgacem, H. I/O Buffer Modelling for Power Supplies Noise Induced Jitter under Simultaneous Switching Outputs (SSO). In Proceedings of the 2019 IFIP/IEEE 27th International Conference on Very Large Scale Integration (VLSI-SoC), Cuzco, Peru, 6-9 October 2019; pp. 226-227.

8. Badaroglu, M.; Wambacq, P.; Van der Plas, G.; Donnay, S.; Gielen, G.G.E.; De Man, H.J. Digital ground bounce reduction by supply current shaping and clock frequency Modulation. IEEE Trans. Comput.-Aided Des. Integr. Circuits Syst. 2005, $24,65-76$.

9. Chung, D.; Ryu, C.; Kim, H.; Lee, C.; Kim, J.; Bae, K.; Yu, J.; Yoo, H.; Kim, J. Chip-package hybrid clock distribution network and DLL for low jitter clock delivery. IEEE J. Solid-State Circuits 2006, 41, 274-286. [CrossRef]

10. Van Toan, N.; Minh Tung, D.; Lee, J. Analysis of Clock Scheduling in Frequency Domain for Digital Switching Noise Suppressions. IEEE Trans. Very Large Scale Integr. (VLSI) Syst. 2018, 26, 1685-1698. [CrossRef] 
11. Wu, T.-L.; Chen, S.-T.; Hwang, J.-N.; Lin, Y.-H. Numerical and experimental investigation of radiation caused by the switching noise on the partitioned DC reference planes of high speed digital PCB. IEEE Trans. Electromagn. Compat. 2004, 46, 33-45. [CrossRef]

12. Pak, J.S.; Kim, H.; Lee, J.; Kim, J. Modeling and Measurement of Radiated Field Emission From a Power/Ground Plane Cavity Edge Excited by a Through-Hole Signal Via Based on a Balanced TLM and Via Coupling Model. IEEE Trans. Adv. Packag. 2007, 30, 73-85. [CrossRef]

13. Wen, S.; Zhang, J.; Lu, Y. Modeling and Quantification for Electromagnetic Radiation of Power-Bus Structure With Multilayer Printed Circuit Board. IEEE Trans. Compon. Packag. Manuf. Technol. 2016, 6, 79-86.

14. Leone, M. The radiation of a rectangular power-bus structure at multiple cavity-mode resonances. IEEE Trans. Electromagn. Compat. 2003, 45, 486-492. [CrossRef]

15. Abhari, R.; Eleftheriades, G.V. Metallo-dielectric electromagnetic bandgap structures for suppression and isolation of the parallel-plate noise in high-speed circuits. IEEE Trans. Microw. Theory Tech. 2003, 51, 1629-1639. [CrossRef]

16. Shahparnia, S.; Ramahi, O.M. Electromagnetic interference (EMI) reduction from printed circuit boards (PCB) using electromagnetic bandgap structures. IEEE Trans. Electromagn. Compat. 2004, 46, 580-587. [CrossRef]

17. Park, J.; Lu, A.C.W.; Chua, K.M.; Wai, L.L.; Lee, J.; Kim, J. Double-stacked EBG structure for wideband suppression of simultaneous switching noise in LTCC-based SiP application. IEEE Microw. Wireless Compon. Lett. 2006, 16, 481-483. [CrossRef]

18. Hassan, M.A.M.; Kishk, A.A. Bandwidth Study of the Stacked Mushroom EBG Unit Cells. IEEE Trans. Antenna. Propagation. 2017, 65, 4357-4362. [CrossRef]

19. Zhang, M.; Li, Y.; Jia, C.; Li, L. Simultaneous Switching Noise Suppression in Printed Circuit Boards Using a Compact 3-D Cascaded Electromagnetic-Bandgap Structure. IEEE Trans. Microw. Theory Tech. 2007, 55, 2200-2207. [CrossRef]

20. Wang, C.-D.; Yu, Y.-M.; Paulis, F.D.; Scogna, A.C.; Orlandi, A.; Chiou, Y.-P.; Wu, T.-L. Bandwidth enhancement based on optimized via location for multiple vias EBG power/ground planes. IEEE Trans. Compon. Packag. Manuf. Technol. 2012, 2, 332-341. [CrossRef]

21. Zhang, M.; Li, Y.; Jia, C.; Li, L. A Power Plane with Wideband SSN Suppression Using a Multi-Via Electromagnetic Bandgap Structure. IEEE Microw. Wireless Compon. Lett. 2007, 17, 307-309. [CrossRef]

22. Park, H.H. Reduction of Electromagnetic Noise Coupling to Antennas in Metal-Framed Smartphones Using Ferrite Sheets and Multi-Via EBG Structures. IEEE Trans. Electromagn. Compat. 2018, 60, 394-401. [CrossRef]

23. Zhang, M.; Li, Y.; Jia, C.; Li, L.; Pan, J. A Double-Surface Electromagnetic Bandgap Structure With One Surface Embedded in Power Plane for Ultra-Wideband SSN Suppression. IEEE Microw. Wireless Compon. Lett. 2007, 17, 706-708. [CrossRef]

24. Kim, M.; Koo, K.; Hwang, C.; Shim, Y.; Kim, J.; Kim, J. A compact and wideband electromagnetic bandgap structure using a defected ground structure for power/ground noise suppression in multilayer packages and PCBs. IEEE Trans. Electromagn. Compat. 2012, 54, 689-695.

25. Kim, M.; Kam, D.G. A Wideband and Compact EBG Structure with a Circular Defected Ground Structure. IEEE Trans. Compon. Packag. Manuf. Technol. 2014, 4, 496-503. [CrossRef]

26. Kim, M. A Compact EBG Structure With Wideband Power/Ground Noise Suppression Using Meander-Perforated Plane. IEEE Trans. Electromagn. Compat. 2015, 57, 595-598. [CrossRef]

27. Kim, Y. Glass-Interposer Electromagnetic Bandgap Structure with Defected Ground Plane for Broadband Suppression of Power/Ground Noise Coupling. IEEE Trans. Compon. Packag. Manuf. Technol. 2017, 7, 1493-1505. [CrossRef]

28. Han, Y.; Huynh, H.A.; Kim, S. Pinwheel Meander-Perforated Plane Structure for Mitigating Power/Ground Noise in System-inPackage. IEEE Trans. Compon. Packag. Manuf. Technol. 2018, 8, 562-569. [CrossRef]

29. Kasahara, Y.; Toyao, H.; Hankui, E. Compact and Multiband Electromagnetic Bandgap Structures with Adjustable Bandgaps Derived From Branched Open-Circuit Lines. IEEE Trans. Microw. Theory Tech. 2017, 65, 2330-2340. [CrossRef]

30. Kasahara, Y.; Toyao, H. Compact (lambda/45 -Sized) Electromagnetic Bandgap Structures with Stacked Open-Circuit Lines. IEEE Microw. Wireless Compon. Lett. 2017, 27, 694-696. [CrossRef]

31. Kamgaing, T.; Ramahi, O.M. A novel power plane with integrated simultaneous switching noise mitigation capability using high impedance surface. IEEE Microw. Wireless Compon. Lett. 2003, 13, 21-23. [CrossRef]

32. Kamgaing, T.; Ramahi, O.M. Inductance-enhanced high-impedance surfaces for broadband simultaneous switching noise mitigation in power planes. In Proceedings of the IEEE MTT-S International Microwave Symposium Digest, 2003, Philadelphia, PA, USA, 8-13 June 2003; Volume 3, pp. 2165-2168.

33. Kim, M. A Miniaturized Electromagnetic Bandgap Structure Using an Inductance-Enhanced Patch for Suppression of Parallel Plate Modes in Packages and PCBs. Electronics 2018, 75, 76. [CrossRef]

34. Lee, J.; Kim, H.; Kim, J. High dielectric constant thin film EBG power/ground network for broadband suppression of SSN and radiated emissions. IEEE Microw. Wireless Compon. Lett. 2003, 15, 505-507.

35. Mohajer-Iravani, B.; Ramahi, O.M. Suppression of EMI and Electromagnetic Noise in Packages Using Embedded Capacitance and Miniaturized Electromagnetic Bandgap Structures with High-k Dielectrics. IEEE Trans. Adv. Packag. 2007, 30, 776-788. [CrossRef]

36. Weiss, J.A. Dispersion and Field Analysis of a Microstrip Meander-Line Slow-Wave Structure. IEEE Trans. Microw. Theory Tech. 1974, 22, 1194-1201. [CrossRef]

37. Agrawal, A.K. Dispersion In n Coupled Microstrip Meanders. IEEE Trans. Microw. Theory Tech. 1980, 28, 927-932. [CrossRef]

38. Yasumoto, K. Coupled-mode formulation of multilayered and multiconductor transmission lines. IEEE Trans. Microw. Theory Tech. 1996, 44, 585-590. [CrossRef] 
39. Sohn, Y.-S.; Lee, J.-C.; Park, H.-J.; Cho, S.-I. Empirical equations on electrical parameters of coupled microstrip lines for crosstalk estimation in printed circuit board. IEEE Trans. Adv. Packag 2001, 24, 521-527. [CrossRef]

40. Bhobe, A.U.; Holloway, C.L.; Piket-May, M. Meander delay line challenge problem: A comparison using FDTD, FEM and MoM. In Proceedings of the 2001 IEEE EMC International Symposium. Symposium Record. International Symposium on Electromagnetic Compatibility (Cat. No.01CH37161), Montreal, QC, Canada, 13-17 August 2001; Volume 2, pp. 805-810.

41. Lee, H.; Kim, J. Unit cell approach to full-wave analysis of meander delay line using FDTD periodic structure modeling method. IEEE Trans. Adv. Packag. 2002, 25, 215-222.

42. Eisenstadt, W.R.; Eo, Y. S-parameter-based IC interconnect transmission line characterization. IEEE Trans. Compon. Hybrid. Manuf. Technol. 1992, 15, 483-490. [CrossRef]

43. Rogers, S.D. Electromagnetic-bandgap layers for broad-band suppression of TEM modes in power planes. IEEE Trans. Microw. Theory Tech. 2005, 53, 2495-2505. [CrossRef] 